\title{
CLAUDIN-3 Protein Expression Relation with Dermatological Diseases: Review Article
}

\author{
Shorouk Hegazy Abdel Hamid*, Ayman ElSayed, Hagar Awad
}

Dermatology, Venereology and Andrology Department, Faculty of Medicine, Zagazig University.

*Corresponding Author: Shorouk Hegazy Abdel Hamid, Email: shoroukhegazy23@gmail.com

\begin{abstract} Claudin-3 level may be altered in patients with psoriasis.

Keywords: CLAUDIN-3 protein, Psoriasis.

\section{INTRODUCTION}

Claudins are a group of transmembrane proteins found in metazoans, particularly in vertebrates and tunicates that help build barriers and pores. Chordate claudins are critical structural proteins in epithelial and endothelial cells' apical junctional complexes where they form tight junction threads (1). In tissues, the claudin protein complexes found in junctional strands serve primarily as paracellular seals for big molecules and as an ion semipermeable barrier. To provide tissuespecific permeability and barrier properties, different claudin members are overexpressed in a given tissue ${ }^{(2)}$.

For the assembly of cell-to-cell signals, claudins act as scaffold proteins. Understanding the expression, organization, and regulation of the claudin family of proteins in the cell at a temporal and geographical resolution is crucial for uncovering processes that affect paracellular barrier function, tissue integrity, and stability ${ }^{(3)}$.
\end{abstract}

Background: When it comes to barrier and pore creation in metazoans, especially vertebrates and tunicates. Claudins are an important family of transmembrane proteins. In epithelia and endothelia's apical junctional complex, chordate claudins are crucial architectural proteins for tight junction strands. Psoriasis patients often have some degree of subclinical intestinal irritation. In gastrointestinal mucosal biopsies, lymphocytic infiltration is found in about $60 \%$ of patients. Intestinal absorption is hampered by structural and functional changes in the colon caused by inflammatory cells. Those who have psoriasis have abnormalities in fecal fat excretion, decreased D-xylose absorption, and impaired lactase activity.Objective: To assess the relation between CLAUDIN-3 protein with dermatological diseases.

Conclusion: Claudin-3 is a key component of tight junctions, which may serve as marker of gut barrier integrity.

\section{Claudins Structure:}

The primary role of epithelial and endothelial cells in most living organisms is organ protection and homeostasis maintenance. Cells' protective barriers are made up of three types of structures: desmosomes, adhesion junction (AJ) cells, and tight junction (TJ) cells ${ }^{(4)}$. Occluding junctions, also called TJs, are multiprotein complexes that help cells keep their barriers intact while simultaneously allowing for intercellular communication and movement. TJs are most frequently seen in the epithelial cell's apical region. TJs have three key transmembrane proteins: junction adhesion molecule (JAM), occludin and claudin ${ }^{(5)}$.

These tiny proteins, which have four transmembrane domains and $\mathrm{N}$ - and $\mathrm{C}$-terminal ends in the cytoplasm as well as two extracellular loops, are called claudins. They range in size from $20-34 \mathrm{kDa}$ (Figure 1) ${ }^{(6)}$. Paracellular charge and ion selectivity are regulated by the first extracellular loop, which is located outside the cell. Second extracellular loop is involved in adhesion of claudin-claudin interaction and functions as a receptor for the toxin produced by bacterial infection ${ }^{(7)}$.

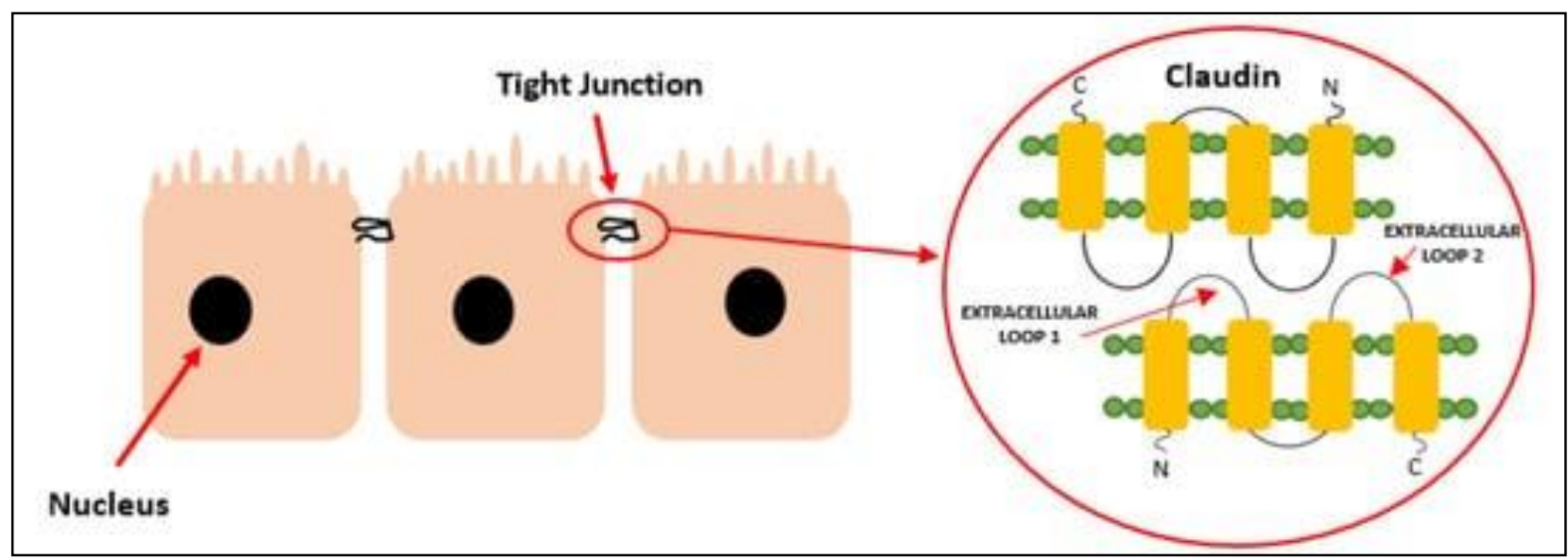

Figure (1): Claudins have a specific structure and function as a component of a tight junction ${ }^{(7)}$

This article is an open access article distributed under the terms and conditions of the Creative Commons Attribution (CC BY-SA) license (http://creativecommons.org/licenses/by/4.0/) 


\section{Claudin-3 expression and regulation:}

There are many distinct types of epithelia, including those of the gastrointestinal system, that express claudin-3. Claudin-3 is found throughout the rat gut, including the jejunum, ileum, and colon, where it is expressed in high levels. However, in the colon, it also demonstrates lateral membrane localization in the colon's crypts and at the surface, demonstrating that it is present in the colon ${ }^{(8)}$.

Prasad et al. ${ }^{\left({ }^{(9)}\right.}$ and Schumann et al. ${ }^{\left({ }^{(10)}\right.}$ found that claudin-3 was concentrated in the tight junctions and lateral cell membranes of human colon crypt and surface epithelial cells.

Claudin-3 has been found to form a barrier in a number of investigations. Using two-path impedance spectroscopy, overexpression of claudin-3 in MDCK II cells increased TER by increasing paracellular resistance and decreasing permeability for both positively and negatively charged ions, as well as bigger molecules like fluorescent dye (332 Da) and FITC-dextran $(4 \mathrm{kDa}){ }^{(11)}$.

It was shown that when claudin-3 was produced in human airway cells (IB3.1), it lowered the paracellular permeability of dextrans of various sizes $(10,70,2000 \mathrm{Da})$ and caused a slight statistically insignificant increase in resistance across the cell layer. Inhibition studies provided more proof of its barrier-forming abilities. In MKN28 gastric epithelial cells, claudin-3 siRNA knockdown resulted in reduced barrier function. Claudin-3 was down regulated in human colon cancer cell lines Caco-2 and $\mathrm{HC} 11$ in response to ochratoxin A and TRPV4, respectively ${ }^{(\mathbf{8})}$.

\section{Claudin 3 serum level in Psoriasis:}

Psoriasis patients often have some degree of subclinical intestinal irritation. In gastrointestinal mucosal biopsies, lymphocytic infiltration is found in about $60 \%$ of patients. Inflammatory cells cause structural and functional gut abnormalities that reduce absorption. Those who have psoriasis have abnormalities in fecal fat excretion, altered D-xylose absorption, and decreased lactase activity ${ }^{(\mathbf{1 2})}$.

Patients with psoriasis have an altered gut microbiome, known as dysbiosis, in addition to inflammation of the intestinal wall. Direct adhesion and the release of metabolites by the intestinal microbiota interact continually with enterocytes ${ }^{(13}$, 14). The establishment of an intestinal barrier is facilitated by physiological colonization of the gastrointestinal tract, whereas dysbiosis interferes with this process and raises gut permeability. Immune activation occurs as a result of bacterial, microbial toxin, and metabolite translocation into the peripheral circulation. Tight connections connect the epithelial cells that make up the gastrointestinal epithelial barrier ${ }^{(15,16)}$.
It's found that those with chronic inflammatory disorders, such as arthritis, celiac disease, and obesity, as well as those with type 2 diabetes, high blood pressure (hypertension), obstructive sleep apnea, and depression, have disruptions in the intestinal barrier ${ }^{(\mathbf{1 7})}$.

The claudins are proteins that attach to actin filaments in the cytoskeleton to help generate tight connections. Loss of gut barrier function is caused by knocking down particular claudins. Claudin3 is a biomarker for intestinal permeability that can be found in the blood ${ }^{(8)}$.

Humbert et al. ${ }^{(18)}$ found that individuals with psoriasis had considerably higher 24-hour urine output of 51Cr-labeled ethylenediaminetetraacetate (51Cr-EDTA) than healthy controls in a study of 15 patients with psoriasis.

Brown et al. ${ }^{(19)}$ reported a series of five case reports, each of which was unique.

The lactulose/mannitol test value was higher in psoriasis patients than in healthy controls. In research, the idea that psoriasis inflammation disrupts the gastrointestinal barrier was confirmed. However, the small number of participants in the listed studies is a serious drawback. This disadvantage may be due to the tests' timeconsuming and complicated technique, which relies on exposing patients to chemicals that are poorly absorbed from their intestines and then collecting their daily urine ${ }^{(\mathbf{1 2})}$.

An accessible and non-invasive diagnostic based on single blood samples is therefore required in every day clinical practice to indicate intestinal permeability with sufficient reliability. Be aware that different aspects of the intestinal barrier may be impacted depending on the condition. There is enhanced gut permeability to bacterial metabolites in an experimental model of hypertension due to diminished colon mucosa, but there are no substantial changes in the tight junction protein ${ }^{(20)}$.

This possible marker for detecting disruption to the intestinal tight junction appears to be Claudin3 because of its modest size, paracellular distribution, and widespread expression in the jejunum and colon. Celiac disease is associated with abnormalities in the intestinal protein claudin-3, a sign of increased intestinal permeability ${ }^{(21)}$.

Patients who engage in strenuous physical activity or have significant surgery may be at risk for congenital cardiac disease. The lactulose-mannitol ratio is associated with plasma claudin-3 concentrations ${ }^{(22)}$.

One plasma marker of tight junction construction and function, zonulin, was studied in individuals with psoriasis because no additional markers were available. Zonulin was found in the blood, although the amount detected $(8.6 \mathrm{ng} / \mathrm{mL})$ was below the detection threshold. This finding could be 
linked to the wide range of zonulin concentrations, some of which may be influenced by inflammation or obesity in humans ${ }^{(23)}$.

Another finding from a prior study found a link between plasma concentrations of a gut integrity dysfunction marker and both the severity of psoriasis and smoking. When it comes to psoriasis, smoking has been linked to an increased risk of the disease developing poorly and responding poorly to therapy (24).

Tobacco use is linked to impaired gut barrier function in several ways. These include altered mucosal immune response, the generation of reactive oxygen species, and changes in the microbiota element of intestine. As a result of these findings, there is now more evidence linking smoking to psoriasis via the intestinal barrier ${ }^{(7,25)}$.

According to the findings of the Sikora et al. (12) study, patients with psoriasis had increased intestinal permeability as measured by plasma concentrations of claudin-3. Smoking and high disease activities are both related with increased intestinal permeability.

These findings demonstrate the significance of the intestinal barrier in the development of psoriasis. New approaches for reducing the onset or exacerbation of psoriasis could be developed by better understanding the interaction between bacteria, the gut barrier, and inflammation ${ }^{(\mathbf{1 2})}$.

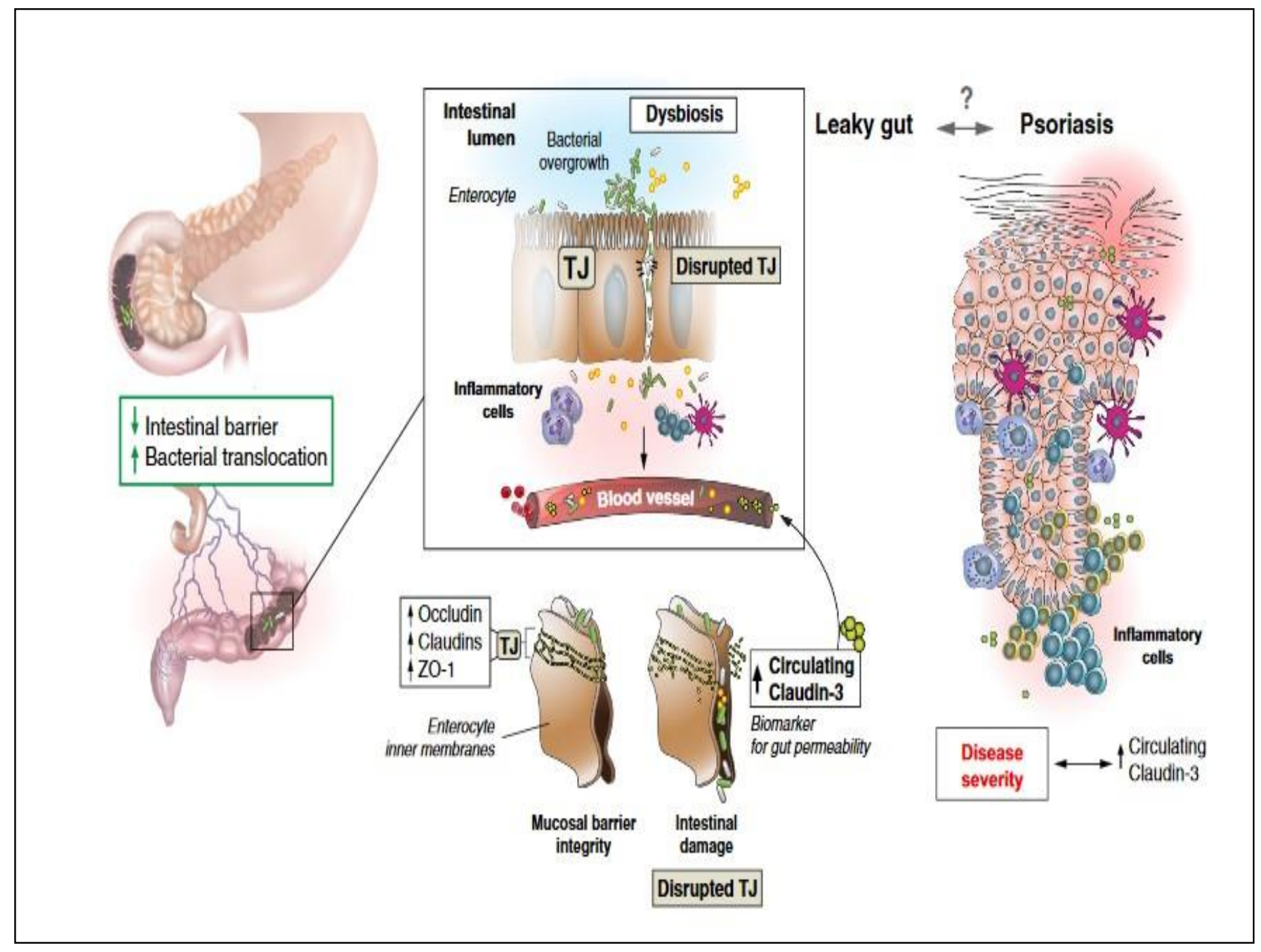

Figure (2): Intestinal barrier disturbance and severe psoriasis may be linked pathogenetically. 
Because of the systemic inflammation caused by gut dysbiosis, the intestinal barrier becomes damaged in psoriasis patients, as seen by a rise in claudin-3 levels in the blood. As a result, the gut mucosa's permeability rises. Transfer of bacteria and their metabolic products into the blood increases the inflammatory response and may worsen the condition of psoriasis ${ }^{(\mathbf{1 2})}$. Claudin-3 levels were found to be considerably greater in individuals with psoriasis in research by Ahmed et al. ${ }^{(26)}$ in Egypt than in healthy controls. Claudin-3 levels were found to be associated with PASI. Claudin-3 levels were found to be considerably greater in smokers. Additionally, as the degree of the injury grew, so did the level of claudin-3. According to Sikora et al. ${ }^{(12)}$ results of a multivariate linear regression study, claudin 3 is linked to the PASI score and active smoking. A substantial association was found between claudin-3 levels and PASI scores, but not between claudin-3 levels and any of the other variables studied. Additionally, they discovered a positive link between claudin 3 concentration and the PASI score. They also discovered that there was no association between BMI and claudin 3 concentrations ${ }^{(12)}$. Smoking and a greater claudin-3 level were found to be substantially related with the development of psoriasis in a univariate study. However, in the multivariate analysis, only a greater level of claudin-3 was found to be an independent predictor of the development of psoriasis. Oral epithelial dysplasias (OED) of various grades exhibit different levels of claudin-1 expression, which has been discovered in previous studies as well (OED). Hämäläinen et $\boldsymbol{a l} .{ }^{\text {(27) }}$ found that in mild and moderate OED, claudin-1 was exclusively found on the cell membrane, whereas in severe OED, it was also found in the cytoplasm. Claudin1 expression in the intermediate epithelial layer was reduced in moderate and severe OED, indicating a function for claudin-1 in the evolution of oral dysplasia (27).

\section{CONCLUSION}

Claudin-3 is a key component of tight junctions, which may serve as marker of gut barrier integrity. Claudin-3 level may be altered in patients with psoriasis.

\section{Financial support and sponsorship: Nil. Conflict of interest: Nil.}

\section{REFERENCES}

1. Van Itallie C, Anderson J (2014): Architecture of tight junctions and principles of molecular composition. In Seminars in Cell Developmental Biology, 36: 157-165.

2. Günzel D, Fromm, M (2012): Claudins and other tight junction proteins. Comprehensive Physiology, 2 (3): 1819-1852.

3. Zihni C, Balda M, Matter K (2014): Signalling at tight junctions during epithelial differentiation and microbial pathogenesis. Journal of Cell Science, 127 (16): 3401-3413.

4. Bhat A, Uppada S, Achkar I, Hashem S et al. (2019): Tight junction proteins and signaling pathways in cancer and inflammation: a functional crosstalk. Frontiers in Physiology, 9: 1942-46.

5. Tabariès S, Siegel $P$ (2017): The role of claudins in cancer metastasis. Oncogene, 36 (9): 1176-1190.

6. Gonçalves A, Ambrósio A, Fernandes R (2013): Regulation of claudins in blood-tissue barriers under physiological and pathological states. Tissue Barriers, 1 (3): 2482-85.
7. Lee B, Kang H, Lee D et al. (2016): Claudin-1,-2,-4, and-5: comparison of expression levels and distribution in equine tissues. Journal of Veterinary Science, 17 (4): 445-49.

8. Barmeyer C, Fromm M, Schulzke J (2017): Active and passive involvement of claudins in the pathophysiology of intestinal inflammatory diseases. Pflügers Archiv-European Journal of Physiology, 469 (1): 15-26.

9. Prasad S, Mingrino R, Kaukinen K et al. (2005): Inflammatory processes have differential effects on claudins 2, 3 and 4 in colonic epithelial cells. Laboratory investigation, 85(9): 1139-1162.

10. Schumann M, Günzel D, Buergel N et al. (2012): Cell polaritydetermining proteins Par-3 and PP-1 are involved in epithelial tight junction defects in coeliac disease. Gut, 61 (2): 220-228.

11. Krug S, Günzel D, Conrad $M$ et al. (2012): Claudin-17 forms tight junction channels with distinct anion selectivity. Cellular and Molecular Life Sciences, 69(16): 2765-2778.

12. Sikora M, Chrabąszez M, Waśkiel-Burnat A et al. (2019): Claudin- 3-a new intestinal integrity marker in patients with psoriasis: association with disease severity. J Eur Acad Dermatol Venereo.1, 33 (10): 1907-1912.

13. Chen Y, Ho H, Tseng $C$ et al. (2018): Intestinal microbiota profiling and predicted metabolic dysregulation in psoriasis patients. Exp Dermatol., 27: 1336-1343.

14. Lin L, Zhang J (2017): Role of intestinal microbiota and metabolites on gut homeostasis and human diseases. BMC Immunol., 18: 2-6.

15. Rooks M, Garrett W (2016): Gut microbiota, metabolites and host immunity. Nature Reviews Immunology, 16 (6): 341-352.

16. Ufnal M, Pham K (2017): The gut-blood barrier permeability - a new marker in cardiovascular and metabolic diseases? Med Hypotheses, 98: 35-37.

17. Liu L, Zhu G. (2018): Gut-brain axis and mood disorder. Frontiers in Psychiatry, 9: 223-26.

18. Humbert P, Bidet A, Treffel $P$ et al. (1991): Intestinal permeability in patients with psoriasis. Journal of Dermatological Science, 2 (4): 324-326.

19. Brown A, Hairfield M, Richards D et al. (2004): Medical nutrition therapy as a potential complementary treatment for psoriasis-five case reports. Alternative Medicine Review, 9 (3): 297-307.

20. Jaworska K, Huc T, Samborowska E et al. (2017): Hypertension in rats is associated with an increased permeability of the colon to TMA, a gut bacteria metabolite. PloS One, 12 (12): 1810-14.

21. Rauhavirta T, Lindfors K, Koskinen $O$ et al. (2014): Impaired epithelial integrity in the duodenal mucosa in early stages of celiac disease. Translational Research, 164 (3): 223-231.

22. Typpo K, Larmonier C, Deschenes J et al. (2015): Clinical Characteristics Associated with Post-Operative Intestinal Epithelial Barrier Dysfunction in Children with Congenital Heart Disease. Journal of the Society of Critical Care Medicine, 16 (1): $37-42$.

23. Tripathi A, Lammers K, Goldblum S et al. (2009): Identification of human zonulin, a physiological modulator of tight junctions, as prehaptoglobin-2. Proceedings of the National Academy of Sciences, 106 (39): 16799-16804.

24. Pezzolo E, Naldi L (2019): The relationship between smoking, psoriasis and psoriatic arthritis. Expert Review of Clinical Immunology, 15 (1): 41-48.

25. Berkowitz L, Schultz B, Salazar G et al. (2018): Impact of cigarette smoking on the gastrointestinal tract inflammation: opposing effects in Crohn's disease and ulcerative colitis. Frontiers in Immunology, 9: 74-79.

26. Ahmed S, Gadalla L, Elmeadawy S et al. (2018): The efficacy of Aloe vera gel in treatment of oral lichen planus. International Dental Medical Journal of Advanced Research, 4 (1): 1-6.

27. Hämäläinen L, Soini Y, Pasonen-Seppänen S et al. (2019): Alterations in the expression of EMT-related proteins claudin-1, claudin-4 and claudin-7, E-cadherin, TWIST1 and ZEB1 in oral lichen planus. Journal of Oral Pathology Medicine, 48 (8): 735744. 